\title{
Partitioning the $N$-space into collinear sets of orthans
}

\author{
Mihály Hujter
}




\title{
PARTITIONING THE $N$-SPACE INTO COLLINEAR SETS OF ORTHANTS
}

\author{
Mihály Hujter \\ Department of Applied Mathematics, University of Miskolc \\ 3515 Miskolc - Egyetemváros, Hungary \\ mathm@gold.uni-miskolc.hu
}

[Received June 29, 2000]

Dedicated to my father on his seventieth birthday.

\begin{abstract}
How many $n$-orthants can be intersected in the $n$-dimensional Euclidean space simultaneously by a single straight line? In this sense of collinearity, how can we partition the entire space into collinear sets of $n$-orthants? How could it happen that a nice and innocent-looking conjecture is false in high dimensions? How are these questions related to the dominating sets in graph theory? What is the relationship to the illumination theory in intuitive geometry? We study such questions in this interdisciplinary paper.
\end{abstract}

Mathematical Subject Classification: 05C69, 52B11, 52C17, 52C35

Keywords: dominating sets in graphs, $n$-dimensional polytopes, packing and covering in $n$-dimensions, arrangements of flats

\section{Introduction}

We consider the $n$-dimensional Euclidean space, denoted $R^{n}$, for $n=2,3, \ldots$ The elements of such a space are the $n$-dimensional columns vectors $x$ with real number components $x_{1}, \ldots, x_{n} \in R$. Another name of such a vector is point. By a sign vector we mean such a vector $s \in R^{n}$ for which each $\left|s_{i}\right|=1$. In other words, the set of the sign vectors is $\{-1,+1\}^{n}$. Obviously, the number of different sign vectors is $2^{n}$. If $s$ is a sign vector, we associate with $s$ the following set, called open $n$-orthant: $\left\{x \in R^{n}: x_{1} s_{1}>0, \ldots, x_{n} s_{n}>0\right\}$. Note that the 2-orthants are known as quadrants, and the 2-quadrant associated to $\left(\begin{array}{c}+1 \\ +1\end{array}\right)$ was named the first quadrant by Monge when he invented descriptive geometry and pioneered the development of analytical geometry two centuries ago. Obviously, the open $n$-orthants are pairwise disjoint open convex sets in $n$ dimensions, and their number is $2^{n}$. Each such open $n$-orthant contains exactly one sign vector, the one that the open $n$-orthant is associated with.

By a straight line in general position we mean a set of vectors $x \in R^{n}$ in the equation form $x=a+t v$ where $t$ runs in $R$ and $a, v \in R^{n}$ are given fixed vectors such that each $a_{i} \neq 0$ and each $v_{i} \neq 0$, furthermore for $i=1,2, \ldots, n$ the unique solutions $t_{i} \in R$ of the equations $0=a_{i}+t_{i} v_{i}$ are all distinct. Geometrically speaking, a straight line in general position is such a line which is neither parallel to the 
coordinate hyperplanes (these are the $n$ hyperplanes with equations $x_{i}=0$, where $i$ is one of $1,2, \ldots, n)$, nor goes through two coordinate hyperplanes at the same point. For example, in 2 dimensions the straight lines in general position are those whose equations are $\alpha_{1} x_{1}+\alpha_{2} x_{2}=\beta$ for $\alpha_{1}, \alpha_{2}, \beta \in R, \alpha_{1} \alpha_{2} \beta \neq 0$.

Obviously, in $n$ dimensions almost all straight lines are in general position. If a straight line is given in the equation form $x=a+t v$ where $t$ runs in $R$ and $a, v \in R^{n}$ are fixed, then obviously, at least one component of $v$ is nonzero. We can change all components $a_{i}$ and $v_{i}$ a little bit for $i=1, \ldots, n$ such a way that the new values all become nonzeros and the $t_{i}$ numbers determined by the equations $0=a_{i}+t_{i} v_{i}$ all become distinct. In this sense any straight line is a "point of accumulation" to the set of the straight lines in general position. It is also obvious, that if an arbitrary straight line meets (i.e., intersects) an arbitrary open $n$-orthant, then by changing the $a_{i}$ and $v_{i}$ components just a little bit we gain a straight line in general position that meets the same open $n$-orthant. In other words, for any fixed $n$-orthant any straight line (not necessarily in general position) which meets the $n$-orthant in question is a "point of accumulation" to the set of straight lines which are in general position and meet the $n$-orthant in question. As a corollary, if each open $n$-orthant in a subset of the set of all open $n$-orthants is met simultaneously by an arbitrary straight line, then a straight line in general position can also be found to meet each element of the subset.

A straightforward question is this: Given a straight line in general position, how many open $n$-orthants can it intersect? Equivalently, how many $n$-orthants can be shot down with one shot from a point $a$ into direction $v$ ? "How many birds can be killed with one stone?" In 2 dimensions the answer is easy because any straight line in general position meets all but one open quadrants. If, for example, $a$ is in the first open quadrant, and $v$ is, for example, in the second open quadrant (the open quadrant associated with $\left({ }_{-1}^{+1}\right)$ ), then the straight line in the form $x=a+t v$ is going to intersect the first, the second and the fourth open quadrants (these are the 2-orthants associated with $\left(\begin{array}{c}+1 \\ +1\end{array}\right),\left(\begin{array}{c}+1 \\ -1\end{array}\right)$, and $\left.\left(\begin{array}{c}-1 \\ +1\end{array}\right)\right)$.

In the next section we will answer the above question in $n$ dimensions. Our Proposition 1 will state that any straight line in general position meets the same number of open $n$-orthants, and this number is exactly $n+1$. From the above argument and from the proof of this proposition it easily follows that in case of a straight line not in general position this number must be smaller, moreover the set of those open $n$-orthants which are met by a straight line not in general position is always a proper subset of a straight line in general position.

In the above discussed sense we can talk about collinear sets of open $n$-orthants. Such a set of open $n$-orthants is collinear whose elements can be met simultaneously in a single straight line in general position. It is clear that one or two open $n$-orthants are always collinear. (The empty set is also collinear.) If a subset of the $2^{n}$ open $n$-orthants is not collinear, we say that the subset is noncollinear.

The collinear sets of $n$-orthants have a simple structure if $n=2$. Namely, any set of at most 3 open quadrants is collinear, but the set of all 4 open quadrants is not. In 3 dimensions the situation is a little bit more complicated. By inspection one can see 
that the set of all 8 open 3-orthants can be partitioned into 2 collinear subsets, and there are 12 such partitions. This way we gain 12 pairs of collinear 4 -element subsets, and all collinear 4-element subsets are among them. However, the total number of 4element subsets is $\left(\begin{array}{l}8 \\ 4\end{array}\right)=70$, and so the number of the noncollinear 4-element subsets is 46. Even a 3-element subset is not necessarily collinear; the number of the 3-element subsets is $\left(\begin{array}{l}8 \\ 3\end{array}\right)=56$, and there are 8 noncollinear 3 -element subsets. An example of such a noncollinear 3-element subset is the one whose elements are associated to those sign vectors in 3 dimensions which have exactly one -1 component. Those subsets which have more than 4 elements are all noncollinear. It is worth mentioning that in 2 dimensions the collinear subsets of the $n$-orthants form a matroid but in higher dimensions they do not.

\section{The maximum number of collinear $n$-orthants}

In this section we study the structure of the inclusion-maximal collinear sets of open $n$-orthants.

Proposition 1. A straight line in general position in $R^{n}$ intersects exactly $n+1$ open $n$-orthants.

Proof. We prove by induction on $n$. The case $n=2$ is obvious by inspection. Assume that $n \geq 3$, and that for smaller dimensions the statement has already been proved. Consider, in $n$ dimensions, a straight line in general position given in the form of the equation $x=a+t v$. Consider the largest $t_{i}$ among the $t_{i}$ numbers which were used in the definition of the straight lines in general position. Without loss of generality we may assume that the largest one among the distinct $t_{i}$ values is $t_{n}$. Let $\varepsilon_{n}>0$ be chosen such that all $t_{i}<t_{n}-\varepsilon_{n}, i=1, \ldots, n-1$. Now observe that for $x^{\prime}=\left(x_{1}, \ldots, x_{n-1}\right)^{T}, a^{\prime}=\left(a_{1}, \ldots, a_{n-1}\right)^{T}, v^{\prime}=\left(v_{1}, \ldots, v_{n-1}\right)^{T}$ the equation $x^{\prime}=a^{\prime}+t v^{\prime}$ defines a straight line in general position in $n-1$ dimensions. By the induction assumption, this line intersects exactly $n$ open $(n-1)$-orthants in $n-1$ dimensions. Since $t_{n}$ was the largest among the distinct $t_{i}$ values, in $n$ dimensions the straight line with equation $x=a+t v$ also intersects $n$ open $n$-orthants for $t<t_{n}$. Note that the last components all have the same sign in these open $n$-orthants. For $t=t_{n}$ the point $x=a+t v$ is on the $n$th coordinate hyperplane. However, for $t>t_{n}$, there is only one open $n$-orthant in which all $a+t v$ points are, and this open $n$-orthant is associated with the sign vector $\left(s_{1}, \ldots, s_{n-1},-s_{n}\right)^{T}$ where the sign vector associated with the point $a+\left(t_{n}-\varepsilon_{n}\right) v$ is $\left(s_{1}, \ldots, s_{n-1}, s_{n}\right)^{T}$. So the entire straight line $x=a+t v$ intersects exactly $n+1$ open $n$-orthants.

\section{Hypercubes and the associated graphs}

By the $n$-dimensional hypercube in standard position we mean the convex hull of the set $\{-1,+1\}^{n} \subseteq R^{n}$. The vertices of the cube are the sign vectors, i.e. the elements of $\{-1,+1\}^{n}$. Given two such vertices, their Hamming distance is the number of 
components where the two vertices, as sign vectors, are different. So the famous Manhattan distance is exactly $2^{n}$ times larger that the Hamming distance. The graph of the hypercube has the same vertex set as the hypercube, and the graph edges are those pairs of vertices whose Hamming distance is exactly one. So the geometrical vertices and the graph theoretical vertices coincide. Similarly, the geometrical hypercube edges and the graph edges also coincide. So the number of vertices in the graph is $2^{n}$, and the number of edges is $n 2^{n-1}$. We color the edges of the graph with colors $1,2, \ldots, n$ as follows: an edge gets color $i$ if the vertices of the edge are different in the $i$ th component and only in that one. So for each color there are exactly $2^{n-1}$ edges. Two edges, as line segments with endpoints at the vertices of the edge, are parallel (in the usual geometrical sense) if and only if they have the same color; otherwise the two edges are geometrically perpendicular to each other.

In Figure 1 we show the hypercube graph for $n=5$. The black dots represent the 32 vertices. The 16 horizontal lines represent the edges of color 1 . The 16 vertical lines represent the edges of color 2 . The edges represented by the lines on the diagonals of the big square are all of color 3 . There are 8 such edges. However, there are 8 more edges of color 3 . Those are all among the sides of the central 20-gon. The tangents of these 8 lines are $+2,-2,+1 / 2$, or $-1 / 2$. The remaining 32 edges form 8 deltoids. There are 2 such deltoids in each corner of the figure. The edges of these deltoids are alternatively of color 4 and color 5 in such a way that the almost vertical edges are all of color 4. (The expression "almost vertical" is understood to mean that the tangent of such a line is at least 2 in absolute value. Similarly, the "almost horizontal" lines are those, whose tangents are between 0 and $1 / 2$.

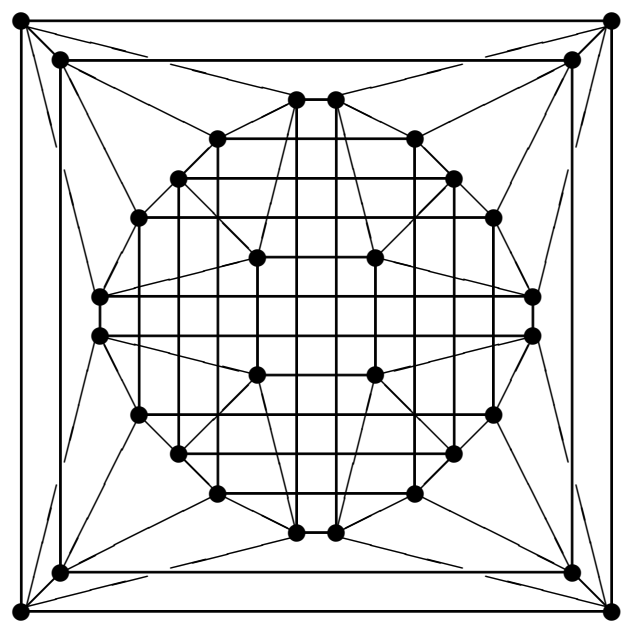

Figure 1. The graph of the 5-dimensional hypercube

We will use some usual notions of graph theory such as a star or a tree. By a super 
tree we mean such a tree, as an induced subgraph in the $n$-dimensional hypercube graph, that has exactly $n$ edges with $n$ different colors. Such a super tree has $n+1$ vertices. By a super star we mean such a super tree which is a star itself. In other words, a super star contains a vertex, called central vertex, and all those vertices, that are neighbors of the central vertex in the graph.

For example, for $n=2$ there are four super trees in the 4 vertex hypercube graph. The vertex sets of these trees can be listed as follows: $\left\{\left(\begin{array}{c}-1 \\ +1\end{array}\right),\left(\begin{array}{l}+1 \\ +1\end{array}\right),\left(\begin{array}{l}+1 \\ -1\end{array}\right)\right\}$, $\left\{\left(\begin{array}{c}+1 \\ +1\end{array}\right),\left(\begin{array}{c}+1 \\ -1\end{array}\right),\left(\begin{array}{c}-1 \\ -1\end{array}\right)\right\},\left\{\left(\begin{array}{c}+1 \\ -1\end{array}\right),\left(\begin{array}{c}-1 \\ -1\end{array}\right),\left(\begin{array}{l}-1 \\ +1\end{array}\right)\right\},\left\{\left(\begin{array}{c}-1 \\ -1\end{array}\right),\left(\begin{array}{l}-1 \\ +1\end{array}\right),\left(\begin{array}{c}+1 \\ +1\end{array}\right)\right\}$. All these super trees are super stars.

For $n \geq 3$, not all super trees are super stars. For example, for $n=3$ we have 8 super stars and 12 super trees that are path graphs in the graph theoretical sense. (A super tree is a path if exactly $n-1$ vertices have 2 neighbors.) The larger the value of $n$ the smaller the percentage the super stars represent among the super trees.

\section{The main result}

Our main theorem can be formulated as follows:

Theorem 2. Consider an arbitrary path, with edges of different colors, in the $n$ dimensional hypercube graph. There exists such a straight line in general position that intersects all $n$-orthants that are associated to the vertices of the path.

Proof. We prove by induction on $n$. Without loss of generality we may assume that the given path has $n$ edges (or equivalently, it has $n+1$ vertices) because in any other case we can add extra vertices and edges. Actually we will prove a stronger theorem. Given a straight line in general position in $R^{n}$ in the form $x=a+t v$, consider the distinct real numbers $t_{i}, i=1,2, \ldots, n$, as above. Let $t_{\min }$ and $t_{\max }$ denote the smallest and largest $t_{i}$, respectively. We claim that the straight line in general position may have such parameters for which $a+\left(t_{\min }-1\right) v$ and $a+\left(t_{\max }+1\right) v$ are in the open $n$-orthants associated with the endvertices of the path. Moreover, we can prescribe that which endvertex should corresponds $t_{\max }$.

The case $n=2$ is easy by inspection. We may assume that $n \geq 3$ and that the statement has already been proved in smaller dimensions. Consider one endvertex of the given path. Let it be denoted as $P_{n}$. Let $P_{n-1}$ denote its neighbor in the path. Without loss of generality we may assume that the edge $\left\{P_{n}, P_{n-1}\right\}$ is of color $n$. Also, without loss of generality we may assume that $P_{n}=(+1,+1, \ldots,+1)^{T}$ as a sign vector. In other words, $P_{n}$ is the vertex which in the first open $n$-orthant. Now the other vertices of the graph all have -1 in their last coordinate because color $n$ can never occur among the colors of the path edges. Similarly to the proof of Proposition 1 we can decrease the dimension number by one by cutting off the $n$th coordinates. By the induction hypothesis we gain that there exists at least one straight line in general position in $R^{n-1}$ which line intersects all open $(n-1)$-orthants that are associated with the first $n$ vertices of our path. Consider such a straight line in the equation form $x^{\prime}=a^{\prime}+t v^{\prime}$ where $x^{\prime}=\left(x_{1}, \ldots, x_{n-1}\right)^{T}, a^{\prime}=\left(a_{1}, \ldots, a_{n-1}\right)^{T}, v^{\prime}=\left(v_{1}, \ldots, v_{n-1}\right)^{T}$. By the induction hypothesis we may assume that $P_{n-1}$ is in the same $(n-1)$-orthant 
as $a^{\prime}+\left(t_{\max }+1\right) v^{\prime}$. Now let us fix $\tau_{n} \in R$ arbitrarily such that $\tau_{n}>t_{\max }+2$ and let $v_{n}=1, a_{n}=-\tau_{n}, a=\left(a_{1}, \ldots, a_{n-1}, a_{n}\right)^{T}, v=\left(v_{1}, \ldots, v_{n-1}, v_{n}\right)^{T}$. We can choose such a value of $t_{n}$ for which the $n$-dimensional straight line with equation $x=a+t v$ will be in general position, and for this straight line all requirements are fulfilled. Here $t_{n}$ will be $\tau_{n}$, and thus for $t>t_{n}$ the points $x+t v$ all will be in the open $n$-orthant associated with $P_{n}$. At the same time for $t<t_{n}, t \neq t_{i}, i=1,2, \ldots, n-1$, the points $x+t v$ will be in the open $n$-orthants associated with the vertex $P \neq P_{n}$ of the $n$-dimensional path in question.

\section{Killing all birds with as few stones as possible}

Consider the open $n$-orthants in $n$ dimensions to be birds in the air. We have to kill all birds. A straight line can be considered to be the orbit of a stone. We have shown that one stone can kill $n+1$ birds. How many stones are needed to kill all birds?

Problem 3. How many straight lines in general position can together meet all open $n$-orthants.

Let $\kappa(n)$ denote the minimum number of straight lines in general position that can together meet all open $n$-orthants. In a more general form and by using different terminology Gy. Kiss conjectured the following in [2].

Conjecture 4. (Gy. Kiss [2]) The vertex set of the $n$-dimensional hypercube graph can be covered by using the vertex sets of $\kappa(n)$ super stars.

Here we tell some reasons to convince the reader that this conjecture is a reasonable one. From the results of Kiss [2] it easily follows that there is bijection from the vertex set of the $n$-dimensional hypercube to the set of all open $n$-orthant with the following property: The image of the vertex set of a super star is an $(n+1)$-element collinear set of open $n$-orthants. On the other hand, from the proofs of our Proposition 1 and Theorem 2 we can see that there is a natural correspondence between the superstars and the paths formed by the sign vectors of the $(n+1)$-element collinear sets of open $n$-orthants. Namely, let $P$ an endvertex of the path, and consider such the uniquely determined superstar whose 2 vertices are $P$ and its neighbor in the path in question. So the central vertex of the super star is the only neighbor of $P$ in the path. Furthermore, for any fixed pair of such a path and such a super star, the bijection of Kiss can be chosen such that the image of this super star is exactly the set of those open $n$-orthants who are associated to the vertices of the path in question. Interestingly enough, those pairs of edges which corresponds to each other, always have the same color in the hypercube graph.

Another fact to convince the reader is this: We can easily check that the conjecture of Kiss is valid for $n=2,3$. In both cases the entire set of all open $n$-orthants can easily be partitioned into 2 collinear subsets. On the other hand, the two super stars with central vertices $s$ and $-s$, where each component of $s$ is +1 , obviously cover the entire vertex set of the hypercube graph.

The conjecture is also valid for $n=4$. One can easily see that the minumum 
number of super stars is the required covering which is nothing else but the famous dominating number of the $n$-dimensional hypercube graph. (Cf. [1] for the importance of the dominating number). It is an easy exercise to check that the dominating number of the 4-dimensional hypercube graph is 4 . The total number of the vertices is 16 , and each super star has 5 vertices. So we need at least 4 super stars. Here are the 4 central vertices of a feasible super star covering: $(+1,+1,+1,-1)^{T}$, $(-1,-1,-1,-1)^{T},(+1,+1,+1,+1)^{T},(-1,-1,-1,+1)^{T}$. On the other hand, the following tables show the column vectors of three sets of collinear open 4-orthants (more precisely, the associated sign vectors):

\begin{tabular}{|c|c|c|c|c|}
\hline+1 & +1 & +1 & +1 & -1 \\
\hline+1 & +1 & +1 & -1 & -1 \\
\hline+1 & +1 & -1 & -1 & -1 \\
\hline+1 & -1 & -1 & -1 & -1 \\
\hline
\end{tabular}

\begin{tabular}{|c|c|c|c|c|}
\hline-1 & -1 & -1 & -1 & +1 \\
\hline+1 & +1 & +1 & -1 & -1 \\
\hline+1 & +1 & -1 & -1 & -1 \\
\hline-1 & +1 & +1 & +1 & +1 \\
\hline
\end{tabular}

\begin{tabular}{|c|c|c|c|}
\hline-1 & -1 & -1 & -1 \\
\hline-1 & -1 & -1 & -1 \\
\hline-1 & -1 & -1 & -1 \\
\hline-1 & -1 & -1 & -1 \\
\hline
\end{tabular}

Only 2 sign vectors are missing, but we know that a 2-element set of open $n$ orthants is always collinear. This completes the proof of Conjecture 4 for $n=4$.

Interestingly enough, Conjecture 4 is not valid for $n=5$.

Theorem 5. We have $\kappa(5)=6$, and the vertices of the 5-dimensional hypercube cannot be covered by the vertex sets of 6 superstars.

Proof. Here are the vertices of 6 paths in the 5-dimensional hypercube (only the signs are showed):

\begin{tabular}{|c|c|c|c|c|c|}
\hline-1 & +1 & +1 & +1 & +1 & +1 \\
\hline+1 & +1 & +1 & -1 & -1 & -1 \\
\hline+1 & +1 & -1 & -1 & -1 & -1 \\
\hline+1 & +1 & +1 & +1 & +1 & -1 \\
\hline-1 & -1 & -1 & -1 & +1 & +1 \\
\hline
\end{tabular}

\begin{tabular}{|c|c|c|c|c|c|}
\hline+1 & -1 & -1 & -1 & -1 & -1 \\
\hline-1 & -1 & -1 & -1 & -1 & +1 \\
\hline+1 & +1 & +1 & -1 & -1 & -1 \\
\hline+1 & +1 & -1 & -1 & -1 & -1 \\
\hline-1 & -1 & -1 & -1 & +1 & +1 \\
\hline
\end{tabular}

\begin{tabular}{|c|c|c|c|c|c|}
\hline-1 & -1 & -1 & +1 & +1 & +1 \\
\hline+1 & +1 & +1 & +1 & -1 & -1 \\
\hline-1 & -1 & +1 & +1 & +1 & +1 \\
\hline+1 & +1 & +1 & +1 & +1 & -1 \\
\hline-1 & +1 & +1 & +1 & +1 & +1 \\
\hline
\end{tabular}

\begin{tabular}{|c|c|c|c|c|c|}
\hline-1 & -1 & -1 & -1 & -1 & +1 \\
\hline-1 & -1 & -1 & -1 & +1 & +1 \\
\hline-1 & -1 & +1 & +1 & +1 & +1 \\
\hline+1 & +1 & +1 & -1 & -1 & -1 \\
\hline-1 & +1 & +1 & +1 & +1 & +1 \\
\hline
\end{tabular}

\begin{tabular}{|c|c|c|c|c|}
\hline+1 & +1 & +1 & +1 & +1 \\
\hline-1 & +1 & +1 & +1 & +1 \\
\hline+1 & +1 & -1 & -1 & -1 \\
\hline-1 & -1 & -1 & -1 & +1 \\
\hline-1 & -1 & -1 & +1 & +1 \\
\hline
\end{tabular}

\begin{tabular}{|c|c|c|c|}
\hline+1 & -1 & -1 & -1 \\
\hline-1 & -1 & +1 & +1 \\
\hline-1 & -1 & -1 & +1 \\
\hline-1 & -1 & -1 & -1 \\
\hline-1 & -1 & -1 & -1 \\
\hline
\end{tabular}

On the other hand, it is well-known in graph theory that the so-called dominating number of the 5-dimension hypercube graph is larger than 6 . This fact can be formulated simply as follows: One cannot take 6 vertices from the graph of Figure 1 such that any other vertex is the neighbor of at least one chosen vertex. By using the symmetry of Figure 1 we can easily check that this statement is true. 


\section{Concluding remarks}

We have proved Conjecture 4 for $n=2,3,4$, and we have disproved it for $n=5$. We have not studied the conjecture in the cases $n=6,7, \ldots$ Kiss [2] proved such a result which implies that Conjecture 4 is valid for infinite different values of $n$.

Acknowledgement: The support provided by the Hungarian National Research Foundation (grant T026575) and by the the "FKFP" Research Fund in Hungary, grant 0486/1999, is gratefully acknowledged.

\section{REFERENCES}

1. Foulds, L. R.: Graph Theory Application, Springer, 1992.

2. KISS, Gy.: Illumination problems and codes, Periodica Mathematica Hungarica, 39(13), (1999), 65-71.. 